Service social

\title{
Groupes et organisation communautaire. Les passerelles de l'intervention
}

\section{Jean-Pierre Deslauriers et Monique Bourget}

Volume 46, numéro 2-3, 1997

Groupes - Symposium 1997

URI : https://id.erudit.org/iderudit/706761ar

DOI : https://doi.org/10.7202/706761ar

Aller au sommaire du numéro

\section{Éditeur(s)}

École de service social de l'Université Laval

ISSN

1708-1734 (numérique)

Découvrir la revue

Citer cet article

Deslauriers, J.-P. \& Bourget, M. (1997). Groupes et organisation communautaire. Les passerelles de l'intervention. Service social, 46(2-3), 77-100.

https://doi.org/10.7202/706761ar
Résumé de l'article

Dans cet article, les auteurs définissent les facteurs politiques, économiques et sociaux qui ont influencé le développement de l'organisation communautaire au cours des années 1980. Cependant, alors que ces années étaient reconnues comme étant celles de la démobilisation et de l'individualisation, des indices ont pointé dans la direction contraire : la prolifération des groupes communautaires, le colloque de Victoriaville et la mise sur pied du Regroupement des intervenantes et intervenants en action communautaire. Les nouvelles circonstances appellent néanmoins un changement de pratique qui se traduit par l'utilisation accrue du groupe en organisation communautaire, de même que la repolitisation par le courant d'empowerment Les auteurs proposent les cuisines collectives comme exemple d'une nouvelle pratique de l'organisation communautaire. 


\title{
Groupes et organisation communautaire Les passerelles de l'intervention
}

\author{
Jean-Pierre DESLAURIERS \\ Monique BOURGET
}

Dans cet article, les auteurs définissent les facteurs politiques, économiques et sociaux qui ont influencé le développement de l'organisation communautaire au cours des années 1980. Cependant, alors que ces années étaient reconnues comme étant celles de la démobilisation et de l'individualisation, des indices ont pointé dans la direction contraire : la prolifération des groupes communautaires, le colloque de Victoriaville et la mise sur pied du Regroupement des intervenantes et intervenants en action communautaire. Les nouvelles circonstances appellent néanmoins un changement de pratique qui se traduit par l'utilisation accrue du groupe en organisation communautaire, de même que la repolitisation par le courant d'empowerment. Les auteurs proposent les cuisines collectives comme exemple d'une nouvelle pratique de l'organisation communautaire.

In this article, the authors explore the political, economic and social factors which have influenced the evolution of community organization in the '80s. However, while these years have been synonymous with individualism and conservatism, some indications seem to point to another direction. In this social landscape, the conference of Victoriaville and the following foundation of the Regroupement des intervenantes et intervenants en action communautaire appear as milestones. Moreover, the proliferation of community groups goes in the same direction. However, new circumstances set the tone for the increased use of groups and group work in community organization. The popularity of the concept of empowerment indicates a new approach to politization. At least, the authors propose that the community kitchens are a good example of this new way to practice community organization. 


\section{INTRODUCTION}

Le travail de groupe fait maintenant partie de l'arsenal de l'organisateur communautaire. En effet, il y a peu de projets d'organisation communautaire qui ne fassent appel au travail de groupe. Certains pensent même que le groupe a colonisé l'organisation communautaire! C'est vrai en partie, car de plus en plus d'intervenants sociaux combinent travail de groupe et organisation communautaire. Cependant, pourquoi le groupe est-il devenu si populaire, et quelle utilisation en fait-on en organisation communautaire? Voilà quelques questions auxquelles cet article tente de répondre.

La première partie porte sur le changement de contexte. Les nouvelles conditions sociales entraînées par l'appauvrissement et les nouveaux problèmes sociaux ont nécessité un changement dans la pratique de l'organisation communautaire : organisateurs et organisatrices ne peuvent plus organiser de la même manière qu'il y a vingt ans. Nous proposons donc quelques explications sur le renouvellement des pratiques d'organisation communautaire et sur le rôle que l'utilisation du groupe y joue.

La deuxième partie porte sur l'utilisation du groupe en organisation communautaire et plus précisément sur ce qui le caractérise. II faut se rappeler que les pionniers américains du travail de groupe reliaient groupe et action sociale, ce avec quoi renouent les tenants de l'empowerment. Le courant de l'empowerment, entendu comme l'acquisition de pouvoir dans un but de transformation sociale, permet selon nous de jeter des ponts entre le travail de groupe et l'organisation communautaire.

La troisième partie présente les cuisines collectives comme un exemple de passerelle dans l'intervention'. Le travail fait dans les cuisines collectives illustre une forme que prend l'organisation communautaire actuellement : changement personnel et changement social sont étroitement reliés, et les actions microscopiques peuvent avoir un effet d'entraînement sur un quartier.

1. Cette partie s'inspire d'une recherche évaluative réalisée par Jean-Pierre Deslauriers [en collaboration avec Carole Brisebois] (1997), Les cuisines collectives : l'expérience du CLSC de Hull, Rapport de recherche, Département de travail social, mars, $120 \mathrm{p}$. 


\section{LE CONTEXTE}

Les années 1980 ont été marquantes dans le développement du mouvement communautaire et des formes qu'il a prises. Dans les années 1970 , le mouvement était porté sur les manifestations à grand déploiement : des grandes marches, des assemblées publiques qui remplissaient le Forum de Montréal, des grèves massives dans le secteur public. Le ton était teinté de revendications et de défense des droits. L'accent était mis sur l'organisation politique des quartiers populaires, et les services - ou ce qui s'y apparentait étaient considérés comme une sorte de diversion : à cette époque, il n'y avait pas de place pour les cuisines collectives! Cependant, au tournant des années 1980, le mouvement communautaire s'est aussi transformé. Il est courant de dire que durant cette décennie le courant axé sur la défense de droits et l'action sociale a plafonné.

Que s'est-il passé au juste? Comment s'explique le retrait de l'action sociale? Plusieurs ont été plus rapides à porter le diagnostic qu'à expliquer la situation. D'abord, la situation politique du Québec y a été pour quelque chose. L'élection du Parti québécois en 1976 avait suscité d'énormes espoirs dans le mouvement populaire et syndical, des espoirs tels qu'un parti pouvait difficilement les satisfaire. À l'occasion du référendum de mai 1980, ce même mouvement avait appuyé le parti au pouvoir, les uns avec plus d'enthousiasme que d'autres, mais peu de forces progressistes ne s'étaient pas prononcées en faveur des thèses gouvernementales. La morosité, pour ne pas dire le découragement, a suivi ce qui a été considéré comme l'échec du référendum. II y eut plus, cependant: le retour des idées libérales et le conservatisme ambiant ont porté un dur coup aux aspirations populaires. Devant la montée de la globalisation et de la mondialisation, termes polis sous lesquels se dissimule le capitalisme triomphant, la gauche a jusqu'ici été incapable d'organiser une riposte et de proposer une solution alternative critique et plausible, faisant le pont entre les initiatives locales et la théorie générale.

Cette morosité a été amplifiée par la pire crise économique que le Québec avait vue depuis la " dépression ": des entreprises ont dû fermer leurs portes sans crier gare, des employés ont perdu leur emploi du jour au lendemain, des entreprises centenaires ont cessé leurs activités. Cette déprime économique a coïncidé avec le retour 
d'idées libérales du siècle passé, d'abord en Angleterre et aux ÉtatsUnis, ensuite au Canada et au Québec. Alors que les mesures sociales devaient servir à alléger la misère des plus pauvres, une certaine élite en a profité pour réduire l'aide qui leur avait été accordée. Les néolibéraux caressaient depuis longtemps le projet de sabrer dans les dépenses publiques : le fardeau de la dette leur a simplement servi de prétexte (Hurtubise et Deslauriers, 1995, p. 312)2.

Au cours de cette décennie, des problèmes sociaux nouveaux ont surgi : la pauvreté a repris du terrain, les ménages se sont appauvris et il est apparu ce qu'on a appelé des nouveaux chômeurs. Ce sont des personnes qualifiées qui exerçaient une occupation avant d'être expulsées du marché du travail : on sait que les personnes qui gonflent les rangs des assistés sociaux sont des travailleurs qui ont épuisé leurs prestations d'assurance-chômage et qu'une grande majorité d'entre eux sont capables de travailler et ne demandent qu'à réintégrer le marché du travail. Cependant, les emplois continuent de manquer à l'appel. À l'expulsion du marché du travail de travailleurs qui en faisaient partie s'ajoutent les difficultés d'insertion des jeunes qui ne peuvent y entrer. Outre les problèmes liés au travail, on a vu poindre le sentiment d'insécurité dans les villes, la violence, les difficultés d'assimiler les immigrants, etc.

Indépendamment des problèmes sociaux accrus, on notait un changement qualitatif dans les rapports sociaux. Le mouvement syndical qui avait été à la pointe des revendications populaires dans les deux décennies précédentes pouvait de plus en plus difficilement réconcilier les différents éléments qu'il regroupait. Comme le notait une observatrice attentive du mouvement syndical au milieu des années 1980 :

Le discours unifiant du mouvement ouvrier traditionnel ne peut plus rejoindre de manière efficace des catégories sociales aussi diversifiées : femmes, hommes, jeunes, manœuvres, ouvriers qualifiés, techniciennes et techniciens, cols blancs des secteurs privé et public, chômeurs, chômeuses, sans-emploi, travailleuses et travailleurs précaires. Cependant, les réalités propres à ces différents groupes demeurent mal connues et le risque est grand

2. Certains auteurs (Martin et Savidan, 1994) ont même parlé de la culture de la dette. 
que le décalage entre leurs pratiques quotidiennes et celle du mouvement syndical ne se résorbe pas facilement et de sitôt (Saint-Pierre, 1985, p. 10).

Ce texte écrit il y a une douzaine d'années demeure d'une brûlante actualité ${ }^{3}$. Écrivant sur le mouvement populaire dans le même numéro de revue, un autre auteur déplorait : "Ce qui est vraiment dramatique, c'est le vide politique... Aucun projet politique ne vient donner sens et perspectives aux actions locales » (Roy, 1985, p. 13). Gabriel Gagnon (1989) a décrit les années 1980 comme des années molles qui nous échappaient comme un savon et où nous n'avions prise sur rien.

Dans ce contexte, alors que certains parlaient de démobilisation et d'individualisation, certains signes démontraient pourtant le contraire. Par exemple, on a assisté au cours des années 1980 à la prolifération de groupes de toutes sortes qui étaient loin d'être des groupes de croissance personnelle. C'est donc dire qu'il était faux de penser que les personnes ne se mobilisaient plus; plutôt, elles ne le faisaient plus pour les mêmes motifs ni de la même manière qu'auparavant. Aux grandes manifestations avait succédé une organisation plus près du terrain, mais plus loin des actions à grand déploiement. Même le militantisme s'était modifié en cours de route : on militait moins par sacrifice et davantage par plaisir; on n'acceptait plus de sacrifier sa vie privée sur l'autel de la cause (Favreau, 1985). Cette redéfinition n'était pas étrangère à l'influence du féminisme qui avait mis en lumière l'interdépendance des aspects personnels et politiques.

Au cours des années 1980, un événement a servi de catalyseur, de phare et de symbole : ce fut le colloque de Victoriaville, qui s'est tenu en 1987. En effet, contrairement à ce qui se pensait et se disait, les organisateurs et les participants de cet événement ont été saisis par l'enthousiasme qui s'est dégagé de cette rencontre : aux yeux de tous, il était évident que le mouvement communautaire était vivant et continuait d'avancer. En 1988, dans la foulée de cette rencontre, les intervenantes et intervenants en action communautaire se sont regroupés à l'intérieur du RQIIAC. Par ses congrès et ses publications,

3. Le numéro de mai 1985 avait porté sur le thème de « La gauche au Québec : 15 ans avant l'an 2000 ». Certains articles sont à relire, dont les deux cités. 
ce regroupement a démontré que la pratique de l'organisation communautaire était multidimensionnelle, diversifiée et qu'elle ne pouvait se réduire à un seul modèle de référence dans lequel le marxisme avait voulu l'enfermer.

À notre avis, il faut poser la question de façon encore plus radicale. Rothman et collab. (1995) ont cherché à conceptualiser ce changement dans la dernière édition de leur livre sur l'intervention communautaire. En effet, même si les auteurs conservent leurs trois modèles de référence (action sociale, planning social, développement local), ils ne parlent plus d'organisation communautaire, mais bien d'intervention communautaire où les sphères d'intervention ne sont plus seulement la communauté, mais aussi les organisations et les petits groupes (p. 3). Ensuite, les auteurs proposent une vision de l'intervention communautaire qui fait place aux aspects individuels des problèmes sociaux :

Nous savons que problèmes et systèmes sont interreliés: la société et la communauté peuvent provoquer ou empirer les troubles des individus, et des valeurs faussées ainsi que des comportements nuisibles individuels peuvent porter préjudice au bien commun. Nous montrerons différents modèles de pratiques génériques où sont combinées les possibilités de l'intervention individuelle et de l'intervention communautaire. Nous soulignerons aussi le recours à la macro-pratique pour soutenir la micro-pratique, à la fois en faisant ressortir l'importance de la structure de l'organisation et en offrant un meilleur encadrement de la politique sociale (p. 4).

C'est ainsi que les auteurs ont ajouté dans la nouvelle édition de leur recueil des articles sur l'intégration des modèles de pratique, l'empowermentet le travail de groupe. Quelle que soit la justesse de leurs propositions, la réorientation théorique de ces auteurs traduit leur volonté de conceptualiser une pratique qui est en train d'émerger.

À partir d'un point de vue plus théorique, Rosanvallon (1995) corrobore ici l'expérience des intervenants en organisation communautaire lorsqu'il explique le changement qualitatif que doivent opérer les politiques sociales : “ L'État-providence était bien organisé pour traiter les problèmes de populations relativement homogènes, de groupes ou de classes si l'on veut. II doit maintenant surtout prendre en charge des individus qui se trouvent tous dans des situations qui leur sont particulières » (p. 197). 
Partant du constat que les mesures générales et universelles ne suffisent plus, cet auteur propose une intervention plus individualisée. À son avis, pour s'attaquer au problème du chômage, par exemple, il faut prendre en considération les variables plus fines que sont la biographie, l'histoire personnelle, l'effet de l'enfance, la situation familiale, la trajectoire professionnelle (Rosanvallon, 1995, p. 199-201). II ne s'agit plus seulement de fournir aux personnes des occasions de participation qui leur manquaient $:$ il faut remonter plus loin, parce que les personnes ont été davantage bafouées. Avant de penser à leur revendication politique, il faut d'abord penser à leur revendication à l'existence sociale. L'auteur appuie un peu fortement sur la nécessité d'individualiser les situations particulières, mais il souligne à juste titre qu'il faut prendre conscience des effets du processus de déstructuration sociale sur les membres d'une société.

Car la pratique de l'organisation communautaire dans les années 1990 a peu en commun avec celle des années 1970 : de fait, il y a tout un monde entre l'organisation communautaire pratiquée dans le contexte de l'État-providence, sur fond de prospérité relative et de solidarité héritée du mode de vie rural, et la conjoncture actuelle. Nous retrouvons de plus en plus de quartiers en état de déliquescence : la drogue a transformé la vie urbaine, dans un contexte de crise financière et d'appauvrissement. II ne suffit plus de passer de l'action sociale au développement local : nous travaillons tout simplement avec de nouvelles personnes aux prises avec des problèmes radicalement nouveaux. De ce point de vue, l'intégration sociale est importante et dépasse de loin le cadre de l'intégration sociale par l'économique 4 . En effet, si la crise sociale est aussi profonde que nous le pensons, l'action communautaire qui se développe et qui se pense aujourd'hui doit être très différente de celle que nous avons connue, à la limite aussi différente que les comités de citoyens des années 1960 le furent de la Ligue du Sacré-Cœur des années 1950!

4. Comme le demande avec pertinence Bernard Eme (1996, p. 24), « L'imaginaire de l'insertion, c'est d'être un "sas" vers le marché du travail. Est-ce qu'au fond, à travers ces services de proximité, il ne faut pas explorer autre chose, un autre sens du mot insertion? C'est-à-dire des activités ayant des valeurs qui ne soient pas seulement portées par le travail salarié? " Est-ce qu'au fond il ne faut pas aussi parler de culture? La réponse à cette question est fondamentale. Voir Eme, Inter-action communautaire, hiver 1996. 
Les organisateurs communautaires n'ont pas été insensibles à ces différents courants. Déjà, au milieu des années 1980, ils commençaient à se poser des questions sur leurs stratégies. Faut-il brûler les pancartes?, comme se le demandaient Jean-François René et Jean Panet-Raymond (1984) dans un pamphlet populaire à cette époque. La supression de postes d'organisateurs communautaires au profit de l'embauche d'agents de relations humaines ainsi que le développement d'une pratique dite intégrée y étaient pour quelque chose; ces deux facteurs avaient contribué à l'éclatement de l'organisation communautaire dans les CLSC. Toutefois, il y avait plus.

En effet, à partir du milieu de la dernière décennie, les organisateurs communautaires ont saisi, parfois de façon intuitive, que l'organisation ne pouvait plus se faire sur les mêmes bases qu'auparavant, en dépit de la misère qu'avait entraînée la crise économique du début des années 1980. Ce n'était plus une question de faire ou non de la contestation : les organisateurs ont réalisé qu'ils ne pouvaient plus répondre adéquatement à la diversité et à la complexité des problèmes sociaux en continuant d'exercer leur profession comme ils le faisaient jadis. C'est ainsi qu'à la suite des expériences sur le terrain on en vint à considérer le développement local comme une voie intéressante de l'organisation communautaire; on a remis sur le tapis le travail de quartier, le développement communautaire, le travail à la base, là où les citoyens et citoyennes se sentent partie prenante, acteurs et sujets de leur existence ${ }^{5}$.

C'est dans cet esprit que nous comprenons l'importance qu'a prise le travail de groupe en organisation communautaire. Le fait qu'on parle maintenant de groupes communautaires au lieu de corps intermédiaires et d'associations volontaires n'est pas qu'une subtilité sémantique, mais traduit la nouvelle réalité dans laquelle se débat le mouvement communautaire. Les intervenantes ont réalisé la force des interventions qui conjuguent le personnel et le social. Dans ce contexte, l'organisation communautaire doit se conceptualiser en faisant un usage accru de la psychologie sociale, tout autant que de la sociologie, de la politique ou de l'économie.

5. Les praticiens de l'entraide internationale en sont arrivés à la même conclusion : au lieu de se lancer dans de grands projets, il vaut mieux en démarrer de plus petits, enracinés dans le milieu. 


\section{L'UTILISATION DU GROUPE EN ORGANISATION COMMUNAUTAIRE}

Même si l'on parle beaucoup du travail de groupe en organisation communautaire, il n'en demeure pas moins que celui-ci est souvent méconnu. La cause à défendre ou l'objectif à poursuivre ne suffisent pas : encore faut-il comprendre le fonctionnement d'un groupe, de même que les différents niveaux de motivation des personnes qui le fréquentent. Plusieurs projets d'organisation communautaire échouent tout simplement parce que les organisateurs ne connaissent pas assez le fonctionnement des groupes :

Les petits groupes sont omniprésents dans l'organisation communautaire. On trouve cependant très peu d'information sur le groupe à la fois dans sa théorie et dans sa pratique. On peut ainsi se demander si plusieurs comités ou autres groupes s'écroulent parce qu'on n'accorde pas assez d'attention à d'importants aspects de la structure du groupe ou aux processus groupaux (Bakalinsky, 1995, p. 145).

La formation des intervenants qui lancent des interventions de groupe ne les a pas toujours préparés adéquatement, qu'ils proviennent de l'intervention personnelle ou de l'organisation communautaire.

Malgré les différences, un groupe demeure un groupe, en organisation communautaire comme ailleurs, et tous les groupes partagent plusieurs similitudes. Tout d'abord, tout groupe socialise les participants : participer à un groupe signifie échanger avec les autres, confronter ses propres valeurs aux leurs, discuter de points de vue différents, se conforter dans son point de vue ou adopter celui d'un autre. Tout groupe peut aussi être l'occasion d'une catharsis pour les participants : certains groupes poursuivent clairement cet objectif, d'autres l'intègrent parfois. Tout groupe peut susciter le changement, au sens restreint comme au sens le plus large. Cependant, certaines caractéristiques distinguent l'usage du groupe en organisation communautaire.

Tout d'abord, l'organisation communautaire vise un changement social par l'intermédiaire du travail de groupe : le groupe est le lieu privilégié pour acquérir une meilleure estime de soi-même, mais aussi pour faire l'apprentissage du pouvoir personnel. " La participation à un groupe permet à ses membres de produire les changements qu'ils 
recherchent et d'acquérir une plus grande connaissance, des habiletés nouvelles, une meilleure image d'eux-mêmes, une plus grande capacité d'action, un plus grand pouvoir et un leadership accru " (Lee, 1994, p. 270). Le changement social ne peut se faire sans changement personnel, c'est évident, et une communauté ne peut s'améliorer sans que les individus qui en font partie progressent eux-mêmes comme personnes, comme parents, comme citoyens. Cette autre citation illustre les liens naturels entre l'organisation communautaire et le travail de groupe :

L'idée de base du travail de groupe est que les membres peuvent, à la fois, s'aider eux-mêmes et s'aider les uns les autres, en échangeant des idées, des suggestions et des solutions, en partageant des sentiments et des informations, en comparant des attitudes et des expériences et en développant leurs relations (Heap, 1987, p. 24).

De plus, l'organisation communautaire introduit une distinction fondamentale avec le groupe de croissance personnelle ou de thérapie. Dans un cas, le groupe est au service de la personne dans le processus de changement personnel; dans l'autre, il est au service de la communauté dans le changement social ${ }^{6}$. L'organisation communautaire vise un changement de conditions sociales :

En organisation communautaire, le but du groupe doit être de changer les conditions qui affectent la communauté dans son ensemble. Le groupe acquiert ainsi une valeur sociale, éducative, politique et même thérapeutique (idem).

C'est évidemment une question d'accent, puisque changement personnel et changement social sont interreliés. II faut cependant toujours conserver à l'esprit qu'en organisation communautaire le groupe est non seulement une fin en soi, mais surtout un moyen?

6. Certains suggèrent de ne pas accentuer théoriquement les différences que la pratique fond et confond : comme le souligne Margot Breton, "Therefore, it may be more appropriate for social workers to conceptualize all their practice as personal change and social action oriented, instead of deciding to work either with "social action" groups or with "personal change" groups " (Breton, 1991, p. 106). La question qui se pose n'est donc pas de distinguer entre le changement personnel et le changement social, mais bien d'encourager et de soutenir le changement tout court. C'est un point de vue intéressant.

7. D'une certaine façon, la notion de groupe en organisation communautaire a des points communs avec ce que certains ont défini comme la micropolitique, c'est-à-dire une conception de l'analyse politique qui utilise des concepts de 


\section{Le travail de groupe et l'action sociale}

Les premiers théoriciens américains du travail social de groupe établissaient des liens très clairs entre le groupe et la politique ${ }^{8}$. En effet, plus d'un auteur voyait le groupe comme un microcosme de la société et comme une sorte d'école de la démocratie. De façon générale, on considérait les groupes comme un moyen pour les pauvres de s'organiser et de faire triompher leur point de vue. Ainsi, avant d'être classée comme méthode de l'organisation communautaire, l'action sociale faisait partie intégrante du travail de groupe ${ }^{9}$. Après avoir passé en revue les idées des premiers théoriciens du travail social de groupe, Ben Z. Shapiro conclut : «Bien que leur vision du groupe comme composante de la politique ne soit pas unique et que l'importance qu'ils lui accordent dans leur philosophie politique varie, ces auteurs ont vu un lien étroit entre l'obligation de l'action sociale et le rôle du groupe dans la société " (1991, p. 12).

Cependant, à mesure que la discipline de travail social s'est professionnalisée et institutionnalisée, le travail de groupe dut défendre ses frontières contre l'organisation communautaire, l'administration et le travail de cas. En outre, cette méthode fut influencée par le développement de la dynamique de groupe. Bref, de fil en aiguille, la question politique perdit de son intérêt. Non pas qu'il ne fût plus mention du pouvoir : au contraire, les premiers auteurs de la dynamique de groupe identifièrent les phénomènes de pouvoir et d'influence au sein des différents groupes qu'ils observaient. Dans leur livre classique, Cartwright et Zander (1968) consacrent de nombreuses pages au pouvoir. Cependant, dans leur optique, le pouvoir est analysé d'un point de vue intra-groupal : les auteurs examinent le pouvoir des participants et leur influence les uns sur les autres, mais non leur influence sur leur environnement. À défaut de retenir

niveau individuel ou groupal pour étudier les phénomènes politiques (Kessel, Cole et Seddig, 1970, p. 1). De l'avis de ces auteurs, la micropolitique présente plusieurs avantages : entre autres, ses concepts s'appliquent directement à plusieurs activités politiques mettant en scène des groupes relativement restreints; ils permettent de réduire les questions complexes, de sorte qu'il devient possible d'y répondre.

8. Le travail social de groupe a d'abord pris racine aux États-Unis où il s'est développé avant d'essaimer dans les autres pays industrialisés.

9. Le livre de Vinik et Levin (1991), Social Action in Group Work, est tout entier consacré à ce sujet. 
cet aspect extra-groupal, l'expérience du pouvoir de groupe devient un événement encapsulé, isolé. En organisation communautaire, l'action dépasse les frontières du groupe pour aborder le plan politique. Margot Breton (1992) avance même que c'est le défi des praticiens :

La dimension extra-groupale ou politique de la conscientisation nous est moins familière ou nous sommes moins portés à l'intégrer. Cette dimension n'exige pas seulement que les individus s'identifient à un petit groupe auquel ils ont le sentiment d'appartenir et dans lequel leurs tentatives d'influence résultent en un plus grand sentiment de bien-être personnel. La dimension politique demande aussi que les individus soient solidaires des autres personnes qui vivent la même situation qu'eux; elle demande qu'ils interagissent avec la communauté dont ils sont aussi partie constituante, en tentant, souvent de façon indirecte, d'influencer ses institutions dans le but de provoquer un changement social et d'amener une justice sociale et un bien-être plus grands (p. 265-266).

En cours de route, pour le meilleur et pour le pire, la psychologie s'est substituée à la théorie politique, l'étude des relations interpersonnelles est devenue plus intéressante que l'action sociale :

En cours de route, la centralité du concept de l'action sociale s'est perdue. Et qui plus est, l'importance du groupe dans l'action sociale et dans le changement social s'est également perdue. C'est ce glissement qui explique comment, dans le travail de groupe, on se soit éloigné de l'action sociale (Shapiro, 1991, p. 13).

Semblable évolution ne fut pas le seul fait du travail social de groupe ni du travail social dans son ensemble : une discipline sœur comme la sociologie a suivi pareille trajectoire. En effet, la sociologie se préoccupait beaucoup de problèmes sociaux et de la réforme sociale à ses débuts; à ce moment-là, le conflit social était au cœur des intérêts des sociologues (Coser, 1956). Cependant, à mesure que la discipline s'est institutionnalisée dans l'université et qu'elle s'est développée comme branche de la connaissance, son centre s'est déplacé vers le pôle de l'intégration sociale, de la déviance. L'évolution du travail social de groupe prend place dans une mouvance qui a traversé plusieurs disciplines, allant de la psychologie aux sciences de l'éducation en passant par la sociologie.

L'influence omniprésente de la psychologie dans les sociétés industrielles avancées traduit la préoccupation de nos contemporains 
pour le vécu plutôt que pour la pensée, le senti plutôt que le raisonné. Les personnes veulent participer, connaître, s'engager, et, de ce point de vue, les interactions entre les participants des groupes sont plus attirantes que le travail politique. Certains établissent même une correspondance entre la remontée du groupe et le déclin de la structure sociale actuelle. En effet, nous serions entrés dans une ère où la communauté, jadis fondée sur des relations interpersonnelles, est maintenant dissoute dans les symboles véhiculés par les médias de masse. Ne subsistent que des fractions de classes, des regroupements, des coalitions, des groupes d'intérêt, bientôt démantelés par l'Internet. Ce genre d'observations a poussé Michel Maffesoli à diagnostiquer le retour des tribus (Maffesoli, 1996, B-1). Ce point de vue traduit la difficulté de concevoir la vie sociale en même temps que la nécessité de vivre la socialité à une plus petite échelle. Poussée à l'extrême, cette vision postmoderniste de la société sombre dans un relativisme qui rend impossible la critique, de même qu'une analyse des conditions concrètes et des effets de la structure sociale ${ }^{10}$.

Cela dit, lorsque le travail social de groupe est apparu au Québec, il en était arrivé à sa phase institutionnelle et professionnalisée aux États-Unis et le thème du changement social avait été presque abandonné. Il en fut de même chez nous. Certes, il y eut bien un retour vers l'action sociale au cours des années 1960 à l'occasion de la guerre à la pauvreté; à ce moment-là, le groupe fut de nouveau utilisé comme moyen de changement social. Vers la même période, au Québec, le travail de groupe fut le fer de lance du mouvement d'animation sociale, de même que celui de l'aménagement du territoire, dans la Gaspésie. Malheureusement, cette résurgence fut de courte durée : l'animation comme levier de changement social dériva en une technique d'organisation de réunion, le travail de groupe devint une technique pure et simple employée à toutes les sauces. Bientôt, on vit apparaître le groupe de croissance.

10. Tous ne partagent pas nécessairement la prémisse du postmodernisme, à savoir la transformation radicale du capitalisme. Comme le demande avec acuité cet auteur: "To what extent has the case been made that the changes in capitalism during the past two decades have been so profound as to represent a destruction of modernist society and a disappearance of history and the "social", i.e. the real face-to-face community? " (Brannigan, 1992, p. 1524). De plus, pour une critique des liens entre postmodernisme et organisation communautaire, voir Hurtubise et Deslauriers (1997), p. 19-22. 
Par un retour du balancier, la politisation est revenue sur le tapis avec l'émergence du courant dit de l'empowerment et l'influence du mouvement féministe. Le groupe, de nouveau considéré comme un lieu de pouvoir, se rapproche de l'organisation communautaire. Nous assistons à une sorte de repolitisation du travail de groupe. Bien que cette pratique soit encore minoritaire, elle nous intéresse.

\section{Groupe et empowerment}

Comme n'importe quel concept devenu à la mode, on donne à celui d'empowerment toutes sortes de colorations pour désigner toutes sortes de pratiques, réelles ou souhaitées, allant de la dynamique de groupe à l'action politique (Lee, 1994, p. 209-210). Par exemple, Lorraine Gutiérrez (1995, p. 205) distingue différents niveaux d'empowerment: le niveau macro, où l'objectif est d'augmenter le pouvoir politique; le niveau micro, où il s'agit d'accroître le pouvoir personnel; un niveau mitoyen, où l'empowerment personnel contribue à l'empowerment du groupe et vice versa. Cette définition a le défaut de trop inclure : à vouloir tout intégrer et s'appliquer à toutes les situations, cette notion finit par ne plus rien signifier. De plus, il s'agit d'une perspective individualiste qui passe sous silence les aspects structurels.

D'autres ont ainsi défini l'empowerment: "The ability and capacity to cope constructively with the forces that undermine and hinder coping, [...] the achievement of some reasonable control over one's destiny " (Pinderhugues, 1983, p. 331). Cette définition de l'empowerment est relativement conservatrice et ne diffère guère de la définition traditionnelle du travail de cas. À force d'essayer de démontrer les liens existant entre la tradition du travail social et ce nouveau concept, l'auteur finit par lui enlever toute couleur. Cette autre définition de l'empowerment présente le même travers que la précédente : "C'est la capacité d'influencer les forces qui affectent l'espace vital d'une personne pour son bénéfice et celui des autres " (Pernell, 1985, p. 117).

La variété des définitions illustre l'ambiguïté que véhicule la notion d'empowerment. Est-ce que l'empowerment individuel, groupal ou communautaire est la même sorte d'empowerment? C'est bien de vouloir augmenter le pouvoir sur sa vie, mais comment? Tout seul? La vertu ne se tient pas toujours au milieu. De plus, la 
réflexion, la prise de conscience, l'empowerment individuel ne suffisent pas à eux seuls :

La conscientisation qui n'est pas suivie de la mobilisation ni de l'organisation en vue d'une action peut fort bien se réduire à une version contemporaine du développement de l'insight. L'empowerment vient d'une action organisée qui s'exerce sur les plans socioéconomique, culturel et politique et qui garantit l'accès aux ressources existantes. On ne doit pas le confondre avec la force personnelle issue d'une action intellectuelle et émotive de découverte et d'apprentissage qui se fait en même temps que la conscientisation (Breton, 1991, p. 105) ${ }^{11}$.

Dans la lorgnette de l'organisation communautaire, l'empowerment signifie que le groupe vise à agir dans la sphère sociale et non pas seulement au niveau individuel ou groupal. « L'orientation sociale guide l'empowerment dans sa participation active et responsable dans la sphère sociale ou publique » (Lewis, 1991, p. 28). Nous retenons du courant de l'empowerment que dans la foulée des pionniers américains le groupe est un lieu d'apprentissage du pouvoir dans le but de changer des conditions sociales. Qui dit groupe dit pouvoir et qui dit pouvoir peut dire aussi politique. Cependant, le passage de l'un à l'autre n'est pas automatique. Ce qui nous intéresse ici est le transfert d'expériences et d'habiletés acquises dans le groupe aux niveaux méso-social et macro-social. Dans cette optique, le groupe est un lieu où la personne apprivoise le pouvoir pour s'en servir dans un but de changement social. Car les gens des quartiers appauvris ne changent pas seulement parce qu'ils vivent dans la misère : s'ils ne changent pas, c'est surtout parce qu'ils considèrent les problèmes de leur communauté comme étant inévitables, démesurés, sans solutions. Même s'ils en souffrent, ils en viennent à s'y résigner. En participant au groupe, ils acquièrent une autre vision, des habiletés :

Le fait d'être engagé dans un effort organisé oblige les gens à s'interroger sur la légitimité d'un pouvoir traditionnel solidement établi. Ils commencent à redéfinir leurs propres notions quant à ce qu'ils sont, à leur rôle dans la communauté et au degré d'influence qu'ils peuvent y exercer. À la suite de cette réflexion, les gens seront d'abod sceptiques quant à la possibilité d'apporter des changements significatifs dans leur communauté (Mondros et Berman-Rossi, 1991, p. 206).

11. Lee (1994) adopte semblable position. 
Dans une optique d'empowerment, le groupe peut jouer un rôle très important dans l'organisation communautaire, un rôle que certains auteurs jugent même irremplaçable :

Le groupe peut faciliter l'empowerment par des moyens dont ne disposent pas les autres formes de travail social. En se reportant à l'accent mis par Solomon (1976) sur les habiletés, les membres du groupe peuvent apprendre les uns des autres comment s'informer, comment choisir et mettre en œuvre les stratégies de changement appropriées, de même que comment se défendre contre toutes représailles. Ils peuvent apprendre ces choses à mesure qu'ils apprennent à se servir des groupes et des institutions autour d'eux pour les rendre conformes à leurs besoins. La relation qui existe entre la responsabilisation dans le groupe et celle dans la société en général devrait être mieux perçue par l'intervenant (Garvin, 1997, p. 277).

Ici encore, le travail de groupe ne suffit pas toujours. Par exemple, comme le souligne avec justesse Garvin (1991, p. 74), il faut la plupart du temps le soutien d'organisations extérieures: tout groupe engagé dans une action sociale sait que les institutions peuvent démontrer une grande résistance au changement et qu'elles peuvent aussi soutenir un long siège sans broncher. Une telle résistance dépasse les forces du groupe et doit être prise en considération dans une stratégie de changement.

Cela dit, même si l'empowerment demeure à la fois un objectif à atteindre et une stratégie à utiliser, il faut soumettre ce concept à la critique, comme n'importe quel autre. II faut bien voir dans quel contexte il émerge, quelles valeurs il sous-tend, quelles fonctions il remplit. Dans un contexte où les services sont réduits parce que les citoyens ont été prétendument habitués à compter sur l'aide de l'État, que signifie l'empowerment? "Parler d'empowerment servirait-il à sur-responsabiliser des individus face à leurs problèmes? Serait-ce un concept permettant parfois de nier la pauvreté et son impact sur des individus et des familles? " (Drolet, 1997, p. 57). La question reste ouverte.

\section{LES PASSERELLES DE L'INTERVENTION : UNE ILLUSTRATION}

Les cuisines collectives constituent un exemple d'empowerment. En effet, l'idée des cuisines collectives est d'abord née de besoins 
économiques concrets : comment arriver à s'alimenter ou à nourrir sa famille décemment pendant tout un mois avec des revenus qui se situent plus souvent qu'autrement sous le seuil de la pauvreté? Voilà l'idée qui est à la base des cuisines collectives. Cependant, les apprentissages des participantes aux cuisines collectives dépassent largement ce cadre. C'est ce que démontre la récente étude de Deslauriers (1997) sur les cuisines collectives, où le chercheur a comparé l'intérêt porté par les femmes à la nutrition (acquisition de nouvelles habitudes alimentaires) à celui qu'elles manifestent à l'égard de l'économie (lutte contre l'appauvrissement) et du développement social et personnel (intégration sociale et promotion de l'engagement communautaire).

Un auteur bien connu dans le domaine du travail social de groupe, Heap (1987), accorde beaucoup d'importance à la phase de préparation du groupe. À défaut de bien se documenter sur ce qui s'est fait ailleurs, sur les collaborations possibles, sur les objectifs du groupe, sur les personnes, l'expérience risque d'être plus difficile à réaliser et la mobilisation des membres pourra s'en ressentir. La même remarque s'applique aux cuisines collectives où le recrutement est une tâche très délicate et pourtant si importante. Au terme de notre recherche, il est apparu crucial qu'une personne se charge en permanence du recrutement, que ce soit une professionnelle, une intervenante d'expérience, une bénévole habituée, etc. Ce doit être une préoccupation constante, car le roulement des participantes est assez élevé; ainsi, dans l'étude ci-dessus mentionnée, les femmes participent à la cuisine collective pendant un an en moyenne. II faut donc que quelqu'un se charge de recruter de nouvelles participantes.

Participer aux cuisines collectives est d'abord une démarche éducative où les femmes acquièrent des connaissances, tant sur les coûts réels des aliments que sur les valeurs nutritives des différents groupes d'aliments. De ce point de vue, les premières rencontres sont centrées sur l'information. Les femmes apprennent aussi comment une saine alimentation peut avoir d'heureuses répercussions sur la santé, même si les habitudes alimentaires ne sont pas faciles à changer et que les résistances sont grandes. En échangeant en groupe sur les réactions de leur famille aux différents mets qui leur ont été proposés, les femmes acceptent de discuter de leurs valeurs familiales, racontent une partie de leur vie et partagent leur 
intimité avec d'autres. Bien que les cuisines collectives constituent un groupe très restreint (elles rassemblent quatre ou cinq femmes, rarement plus, avec une personne qui les aidera à s'organiser), leur pratique s'analyse par beaucoup d'aspects à l'aide de la dynamique de groupe.

Comme dans tout groupe nouvellement constitué, les participantes doivent rapidement aborder des questions de fonctionnement (horaire, propreté et entretien des lieux), d'engagement personnel et de distribution de tâches. Très tôt, elles déterminent leurs capacités, leurs compétences et leurs intérêts en s'appropriant certaines tâches. Rapidement aussi, elles sont en mesure de constater l'effet d'un manque d'empressement de l'une ou l'autre à s'acquitter de ses tâches. Voilà autant d'occasions pour ces personnes d'apprendre d'abord à définir les problèmes, à les nommer pour trouver des solutions, à prendre la parole en groupe et à courir le risque de s'exprimer. Et quand des conflits surgissent, le rôle de l'intervenante consiste à revenir sur la notion de contrat du groupe. La structuration de l'action est un rôle très important dans le fonctionnement des cuisines et, de ce point de vue, les intervenantes exercent une influence déterminante.

Cependant, les femmes ne retiennent pas que les connaissances diététiques qu'elles acquièrent en participant aux cuisines collectives : elles aiment surtout se retrouver entre elles, ne plus être seules, pouvoir jaser. De ce point de vue, les notions de réciprocité, d'entraide, d'aide mutuelle sont au cœur du fonctionnement des cuisines :

Lorsqu'une personne exprime ses pensées, ses sentiments, ses émotions, elle témoigne ainsi de la confiance qu'elle accorde à la personne qui l'écoute... Lorsqu'une personne accorde sa confiance à une autre, elle permet à celle-ci de recevoir quelque chose, de s'enrichir, car nous avons tous besoin d'apprendre, d'aimer et d'être aimé... Le fait d'être entendu et reçu est précieux, car chacun a besoin d'être reconnu et aimé (De Luca et collab., 1997, p. 9).

La cuisine collective a aussi des effets sur la culture et sur l'environnement. Par l'entremise de la chaîne téléphonique, chacune est prévenue d'une rencontre imprévue, d'une conférence, d'un stage de formation sur un sujet ou un autre. En plus de briser l'isolement, 
il est possible de créer de nouvelles alliances, de nouveaux contacts, d'établir de nouvelles appartenances, de s'ouvrir à de nouveaux horizons. Ainsi, le changement personnel se transforme peu à peu en action, en nouvelle façon de faire rendue possible d'abord par l'acquisition de nouvelles habiletés.

Ces personnes deviennent souvent des agents multiplicateurs dans leur milieu. " La cuisine m'a ouverte au communautaire ", dira une participante. Plusieurs anciennes participantes aux cuisines collectives dans le quartier étudié ont siégé à différents comités : comité de résidants, comité de parents, comité des loisirs, comité d'organisation de petits-déjeuners communautaires, participation à un comité d'organisation d'un repas multiculturel, comité de la fête du 8 mars, etc. D'autres ont mis sur pied un réseau d'entraide où elles échangent des heures de garde des enfants avec leurs voisines. Dans les quartiers défavorisés, certains parents conservent un très mauvais souvenir de leur court passage à l'école. Pourtant, il nous a été donné d'observer une participante qui a joint les rangs d'un comité de parents à l'école que fréquentait son enfant pendant qu'une autre retournait aux études pour terminer son cours secondaire. Voilà des changements significatifs dont les répercussions se traduisent souvent par un plus grand engagement des femmes dans leur propre communauté.

Sur le plan de la préparation du groupe, le recrutement des participantes aux cuisines collectives pourrait être une occasion pour les intervenantes de solliciter parmi les collègues de travail des noms de personnes intéressées par ce genre de projet. Cela aurait pour effet de briser l'isolement professionnel des intervenantes tout en leur permettant de mettre en commun leurs connaissances tant sur la problématique de la pauvreté que sur la clientèle, le milieu socioéconomique du territoire à desservir, la nature des obstacles ou des contraintes à anticiper. Ces connaissances sont indispensables au bon démarrage d'un groupe. La compétence des intervenantes est mise à contribution non seulement dans le démarrage et le recrutement, mais aussi dans le développement de la réflexion, de la mise en relation des conditions de vie et des structures sociales. C'est à partir d'une telle analyse que le changement social est une avenue non seulement possible mais désirable. 
C'est dans cette optique que nous comprenons la vogue des cuisines collectives : celles-ci représentent une nouvelle forme d'action communautaire qui rejoint les personnes d'une manière adaptée à la conjoncture actuelle. C'est une approche où se conjuguent la réhabilitation personnelle, l'entraide, l'acquisition de connaissances, le fait de passer du niveau microsocial au niveau méso-social par l'intermédiaire des organisations de voisinage. II s'agit d'une intervention où toute la personne est engagée, où les aspects personnels sont importants, où la trajectoire personnelle et inter-générationnelle doit être prise en considération. Ce contexte a remis à l'avant-scène l'importance du travail de quartier et du développement communautaire. C'est un niveau où les citoyens et citoyennes se sentent partie prenante, acteurs et sujets de leur existence : " Le défi actuel consiste à aider à mettre sur pied des réseaux de soutien, au niveau local, ainsi que des réseaux adaptés à la société technologique, en travaillant au niveau des quartiers et des communautés. Et nous pouvons aider les défavorisés à s'aider eux-mêmes à retrouver un sentiment de pouvoir et d'estime de soi dans le processus" (Naparstek, 1997, p. 17).

\section{CONCLUSION}

En conclusion, même si les liens entre l'organisation communautaire et le travail de groupe sont nombreux et évidents, surtout dans une perspective d'empowerment, cette nouvelle approche n'est pas la solution universelle à tous nos maux. Car si l'empowerment est la solution, où est le problème? Cependant, l'importance de ce courant est qu'il attire une fois de plus l'attention sur une question qui est au cœur de l'organisation communautaire et du travail social, soit celle du pouvoir, de la structure sociale et de son influence dans la vie quotidienne.

De plus, le processus d'empowerment peut s'étaler sur plusieurs années. On ne peut penser remettre sur pied, en quelques mois ou même en quelques années, un quartier que la misère a mis plusieurs années à démolir. II faut y mettre du temps. De plus, les progrès sont souvent minimes, difficiles à cerner et lents à venir. "Les actions menant à l'empowerment ne bouleversent rien. Elles ne changent pas l'ordre mondial comme un raz-de-marée " (Lee, 1994, p. 297). 
Hélas, la lenteur du processus ne va pas toujours de pair avec l'empressement des gestionnaires (ou même des praticiens!) à présenter des résultats concrets. Dans l'exemple que nous venons de décrire, les participantes pensaient mettre sur pied une cuisine collective en trois mois : trois ans après, elles y travaillaient encore. Elles pensaient aussi se retirer du quartier après quelques années : les premiers résultats probants mirent huit ans à émerger. Néanmoins, l'expérience nous apprend ceci : lorsque les personnes sont encouragées à évoluer et qu'elles voient des possibilités de changement, elles s'y aventurent tôt ou tard. Lorsque les praticiens sont suffisamment habiles et patients pour définir les obstacles et soutenir ce processus, lorsqu'ils sont suffisamment ouverts pour partager leurs connaissances, leurs habiletés et leur pouvoir, les résultats obtenus dépassent souvent leurs espérances.

Jean-Pierre DESLAURIERS

Professeur

Département de travail social Université du Québec à Hull

Monique BOURGET

Agente de stage

Département de travail social Université du Québec à Hull

\section{Références bibliographiques}

BAKALINSKY, R. (1995). " The small group in community organization practice ", dans J. Rothman, J. Erlich, J.E. Tropman (dir.), (1995), Strategies of Community Organization. Macro practice, $5^{\mathrm{e}}$ édition, Ithasca, Peacock, p. 140-146.

Brannigan, A. (1992). " Postmodernism ", dans Edgar F. Borgatta et M.L. Borgatta (dir.), Encyclopedia of Sociology, New York, Macmillan Publishing, vol. 3, p. 1522-1525.

BRETON, M. (1992). « Liberation Theology, Group Work, and the Right of the Poor and Oppressed to Participate in the Life of the Community ", dans J.A. Garland (dir.), Group Work Reaching Out : People, Places and Power, New York, Haworth Press, p. 257-269. 
BRETON, M. (1991). "Reflections on Social Action Practice in France ", dans A. VINIK et M. LEVIN (dir.), Social Action in Group Work, New York, Haworth Press, p. 91-107.

Calhoun, C. (1992). "Social change ", dans Edgar F. Borgatta et M.L. Borgatta (dir.), Encyclopedia of Sociology, New York, Macmillan Publishing, vol. 4, p. 1807-1812.

CARTWRIGHT, D. et A. ZANDER (1968). "Power and influence in group », dans D. Cartwright et A. Zander (dir.), Group Dynamics, New York, Harper \& Row, p. 215-236.

Corporation de développement communautaire des Bois-Francs (1987). Fais-moi signe de changement, Actes du colloque provincial sur le développement communautaire, Victoriaville.

COSER, L. (1956). The Functions of Social Conflict, New York, Free Press.

DESLAURIERS, J.-P. [avec la participation de C. BRISEBOIS] (1997). Les cuisines collective : l'expérience du CLSC de Hull, Département de travail social, rapport de recherche déposé à la Régie régionale de la santé et des services sociaux de l'Outaouais, mars, $120 \mathrm{p}$.

DROLET, M. (1997). « Empowermentet intervention familiale : concept paradoxal occultant parfois la pauvreté ", Reflets, vol. $3, n^{\circ} 1$, printemps, p. 55-79.

EME, B. (1996). "L'insertion ou comment le "tiers secteur institutionnel" se ferme sur lui-même », Inter-action communautaire, nos 36-37, hiver, p. 24.

FAVREAU, L. (1985). « Militer autrement », Mouvements, mai, p. 24.

GaGnoN, G. (1989). "La complainte des années molles », Possibles, vol. 13, nos 1-2, hiver, p. 19-26.

GARVIN, C.D. (1997). Contemporary Group Work, $3^{e}$ édition, Boston, Allyn and Bacon.

GARVIN, C.D. (1991). « Barriers to Effective Social Action by Groups », dans A. Vinik et M. Levin (dir.), Social Action in Group Work, New York, Haworth Press, p. 65-76.

GUTIÉRREZ, L. (1995). “ Working with women of color : an empowerment perspective ", dans J. Rothman, J. Erlich, J.E. Tropman (dir.), Strategies of Community Organization. Macro practice, $5^{\mathrm{e}}$ édition, Ithasca, Peacock, p. 204-212.

HEAP, K. (19870. La pratique du travail social de groupe, Paris, Éditions ESF.

HUMBERT, C. (1987). "L'enquête conscientisante ", dans J.-P. Deslauriers (dir.), Les méthodes de la recherche qualitative, Sillery, Presses de l'Université du Québec, p. 91-105. 
HUMBert, C. (1976). Conscientisation. Expériences, positions dialectiques et perspectives, Paris, L'Harmattan.

HURTUBISE, Y. et J.-P. DESLAURIERS (1997). « La nouvelle donne de l'organisation communautaire : réponse aux contraintes imposées à la pratique par le néolibéralisme, le postmodernisme et la crise des finances publiques ", Intervention, no 104, mars, p. 16-26.

HURTUBISE, Y. et J.-P. DeslaURIERS (1995). " Perfectionnement des intervenantes et intervenants en CLSC », dans C. Mercier, C. Gendreau, J. Dostie et L. Lafontaine (dir.), Au cœur des changements sociaux : les communautés et leurs pouvoirs, Regroupement des intervenantes et intervenants en action communautaire en CLSC et en centre de santé, Université de Sherbrooke, p. 311-323.

KESSEL, J.H., G.F. COLE et R.G. SeddIG (1970). « Introduction », Micropolitics. Individuals and Group Level Concepts, New York, Holt, Rinehart and Winston, p. 1-15.

LEE, J.A.B. (1994). The Empowerment Approach to Social Work Practice, New York, Columbia University Press.

LEWIS, E. (1991). "Social Change and Citizen Action : A Philosophical Exploration for Modern Social Group Work », dans A. Vinik et M. Levin (dir.), Social Action in Group Work, New York, Haworth Press, p. 23-34.

LUCA, T. de, S. GIASSON, C. PHARAND et C. Ross (1997). L'entr'aide : guide d'accompagnement, Comité d'action promotionnelle de la santé, Hull.

MAFFESOLI, M. (1996). "Michel Maffesoli, prophète de l'écologisation ». Entrevue réalisée par Antoine Robitaille, Le Devoir, 21 octobre, B-1.

MARTIN, P. et P. SAVIDAN (1994). La culture de la dette, Montréal, Boréal.

MONDROS, J. et T. BERMAN-ROSSI (1991). " The Relevance of Stages in Group Development Theory to Community Organization Practice ", dans A. Vinik et M. Levin (dir.), Social Action in Group Work, New York, Haworth Press, p. 203-221.

NAPARSTEK, A.J. (1997). "Community Building and Social Group Work », Social Work with Groups Newsletter, vol. 13, n 1, mai, p. 14-15, 17.

PERNELL, R.B. (1985). « Empowerment and social group work », dans M. PARNES (dir.), Innovations in Social Group Work: Feedback from Practice to Theory, p. 107-117.

PINDERHUGUeS, E.B. (1983). “ Empowerment for Our Clients or Ourselves », Social Casework, 64, p. 331-338.

RENÉ, J.-F. et J. PANET-RAYMOND (1984). Faut-il brûler les pancartes? Le mouvement populaire aujourd'hui, Montréal, Journal La Criée et ACEF du Centre de Montréal. 
Rosanvallon, P. (1995). La nouvelle question sociale. Repenser l'Étatprovidence, Paris, Seuil.

Rothman, J., J. ERLICH et J.E. TROPMAN [dir.] (1995). Strategies of Community Organization. Macro Practice, $5^{\ominus}$ édition, Ithasca, Peacock.

ROTHMAN, J. (1995). "Introduction », dans J. Rothman, J. Erlich et J.E. Tropman (dir.), Strategies of Community Organization. Macro Practice, $5^{\mathrm{e}}$ édition, Ithasca, Peacock, p. 3-25.

RoY, L. (1985). Mouvements, mai, p. 13.

SAINT-PIERRE, C. (1985). "Le difficile chemin du mouvement syndical ", Mouvements, mai, p. 10-11.

SHAPIRO, B.Z. (1991). "Social Action, the Group and the Society ", dans A. Vinik et M. Levin (dir.), Social Action in Group Work, New York, Haworth Press, p. 7-21.

SOLOMON, B. (1976). Black Empowerment, New York, Columbia University Press.

VINIK, A. et M. LEVIN (1991). Social Action in Group Work, New York, Haworth Press. 